TRANSACTIONS OF THE

AMERICAN MATHEMATICAL SOCIETY

Volume 351, Number 11, Pages 4599-4609

S 0002-9947(99)01907-8

Article electronically published on July 21, 1999

\title{
A CLASSIFICATION OF BAIRE-1 FUNCTIONS
}

\author{
P. KIRIAKOULI
}

\begin{abstract}
In this paper we give some topological characterizations of bounded Baire-1 functions using some ranks. Kechris and Louveau classified the Baire- 1 functions to the subclasses $\mathbb{B}_{1}^{\xi}(K)$ for every $\xi<\omega_{1}$ (where $K$ is a compact metric space). The first basic result of this paper is that for $\xi<\omega, f \in \mathbb{B}_{1}^{\xi+1}(K)$ iff there exists a sequence $\left(f_{n}\right)$ of differences of bounded semicontinuous functions on $K$ with $f_{n} \rightarrow f$ pointwise and $\gamma\left(\left(f_{n}\right)\right) \leq \omega^{\xi}$ (where " $\gamma$ " denotes the convergence rank). This extends the work of Kechris and Louveau who obtained this result for $\xi=1$. We also show that the result fails for $\xi \geq \omega$. The second basic result of the paper involves the introduction of a new ordinal-rank on sequences $\left(f_{n}\right)$, called the $\delta$-rank, which is smaller than the convergence rank $\gamma$. This result yields the following characterization of $\mathbb{B}_{1}^{\xi}(K): f \in \mathbb{B}_{1}^{\xi}(K)$ iff there exists a sequence $\left(f_{n}\right)$ of continuous functions with $f_{n} \rightarrow f$ pointwise and $\delta\left(\left(f_{n}\right)\right) \leq \omega^{\xi-1}$ if $1 \leq \xi<\omega$, resp. $\delta\left(\left(f_{n}\right)\right) \leq \omega^{\xi}$ if $\xi \geq \omega$.
\end{abstract}

\section{INTRODUCTION}

Let $K$ be a compact metric space and $C(K)$ the set of continuous real-valued functions on $K$. A function $f: K \rightarrow \mathbb{R}$ is Baire- 1 if there exists a sequence $\left(f_{n}\right)$ in $C(K)$ that converges pointwise to $f$. Let $\mathbb{B}_{1}(K)$ be the set of bounded Baire-1 functions on $K$. Haydon, Odell and Rosenthal in [H-O-R] and Kechris and Louveau in $[\mathrm{K}-\mathrm{L}]$ defined the oscillation rank $\beta(f)$ of a general function $f: K \rightarrow \mathbb{R}$ and proved that $f$ is Baire- 1 iff $\beta(f)<\omega_{1}$. Also, for every ordinal $\xi<\omega_{1}$ the subclass $\mathbb{B}_{1}^{\xi}(K)$ was defined by Kechris and Louveau in $[\mathrm{K}-\mathrm{L}]$ to be the set of all $f$ in $\mathbb{B}_{1}(K)$ such that $\beta(f) \leq \omega^{\xi}$, and it was proved that $f$ in $\mathbb{B}_{1}^{1}(K)$ iff $f$ is the uniform limit of differences of bounded semicontinuous functions on $K$ (Theorem 3 ). Theorem 3 was originally proved in $[\mathrm{H}-\mathrm{O}-\mathrm{R}]$ ( where $\mathbb{B}_{1}^{1}(K)$ is called $\mathbb{B}_{1 / 2}(K)$ ). This is in fact stated in $[\mathrm{K}-\mathrm{L}]$, just before the statement of their Theorem 1 , Section 3.

In this paper we give a general result for $\mathbb{B}_{1}^{\xi}(K)$ which is analogous to the above result for $\mathbb{B}_{1}^{1}(K)$.

In Theorem 7, we obtain the result that for $\xi<\omega, f \in \mathbb{B}_{1}^{\xi+1}(K)$ iff there exists a sequence $\left(f_{n}\right)$ in $\operatorname{DBSC}(K)$ with $f_{n} \rightarrow f$ pointwise and $\gamma\left(\left(f_{n}\right)\right) \leq \omega^{\xi}$ (where " $\gamma$ " denotes the convergence rank, whose definition is recalled below). This extends the work of $[\mathrm{K}-\mathrm{L}]$, who obtained this result for $\xi=1$. We also show in Corollary 9 that the result fails for $\xi \geq \omega$; indeed we obtain there that if $f_{n} \rightarrow f$ pointwise and $\gamma\left(\left(f_{n}\right)\right) \leq \omega^{\xi}$ with $\left(f_{n}\right) \subset \operatorname{DBSC}(K)$, then also $\beta(f) \leq \omega^{\xi}$. Also Proposition 12 shows that Theorem 7 fails if we suppose in addition that $\sup _{n}\left|f_{n}\right|_{D}<\infty$. In Theorem 8 we obtain that if $f_{n} \rightarrow f$ pointwise, with $f_{n}$ 's Baire- 1 functions, $\lambda$ a

Received by the editors July 11, 1994 and, in revised form, December 28, 1995.

1991 Mathematics Subject Classification. Primary 03E15, 04A15, 46B99, 54C50. 
limit ordinal, and $m<\omega$, with $\gamma\left(\left(f_{n}\right)\right) \leq \omega^{\lambda+m}$ and $\sup _{n} \beta\left(f_{n}\right)<\omega^{\lambda}$, then $f$ is Baire-1 with $\beta(f) \leq \omega^{\lambda+m}$. In Proposition 10 we show by example that this result fails, if we allow $\sup _{n} \beta\left(f_{n}\right)=\omega^{\lambda}$ instead (for $\lambda=\omega$ ).

The final result of the paper, Theorem 17, involves the introduction of a new ordinal-rank on sequences $\left(f_{n}\right)$, called the $\delta$-rank, which is smaller than the convergence rank $\gamma$. This is motivated by a characterization of $\mathbb{B}_{1 / 4}(K)$ given in [H-O-R]. Theorem 17 yields the following characterization of $\mathbb{B}_{1}^{\xi}(K)$, analogous to the $\mathbb{B}_{1 / 4}(K)$ characterization given in [H-O-R]: $f \in \mathbb{B}_{1}^{\xi}(K)$ iff there exists a sequence $\left(f_{n}\right)$ of continuous functions with $f_{n} \rightarrow f$ pointwise and $\delta\left(\left(f_{n}\right)\right) \leq \omega^{\xi-1}$ if $1 \leq \xi<\omega$, resp. $\delta\left(\left(f_{n}\right)\right) \leq \omega^{\xi}$ if $\xi \geq \omega$. In fact, such a sequence $\left(f_{n}\right)$ may be chosen as convex blocks of any sequence $\left(g_{n}\right)$ of continuous functions converging pointwise to $f$; the analogous result for the $\gamma$-rank is due to Kechris and Louveau, and used in a fundamental way in the proof.

1. Definition. Let $K$ be a compact metric space, $f: K \rightarrow \mathbb{R}, P \subset K$ and $\varepsilon>0$. Let $P_{\varepsilon, f}^{0}=P$ and for any ordinal number $a$ let $P_{\varepsilon, f}^{a+1}$ be the set of those $x \in P_{\varepsilon, f}^{a}$ such that for every open set $U$ around $x$ there are two points $x_{1}$ and $x_{2}$ in $P_{\varepsilon, f}^{a} \cap U$ such that $\left|f\left(x_{1}\right)-f\left(x_{2}\right)\right| \geq \varepsilon$.

At a limit ordinal $a$ we set

$$
P_{\varepsilon, f}^{a}=\bigcap_{\beta<a} P_{\varepsilon, f}^{\beta}
$$

Let

$$
\beta(f, \varepsilon)=\left\{\begin{array}{l}
\text { the least } a \text { with } K_{\varepsilon, f}^{a}=\varnothing \text { if such an } a \text { exists, } \\
\omega_{1}, \quad \text { otherwise. }
\end{array}\right.
$$

Define the oscillation rank $\beta(f)$ of $f$ by

$$
\beta(f)=\sup \{\beta(f, \varepsilon): \varepsilon>0\} .
$$

The above rank is defined by Haydon, Odell and Rosenthal in [H-O-R] and Kechris and Louveau in $[\mathrm{K}-\mathrm{L}]$.

Let $\left(f_{n}\right)$ be a sequence of real functions on $K, P \subset K$ and $\varepsilon>0$. Let $P_{\varepsilon,\left(f_{n}\right)}^{0}=P$ and for any ordinal number $a$ let $P_{\varepsilon,\left(f_{n}\right)}^{a+1}$ be the set of those $x \in P_{\varepsilon, f}^{a}$ such that for every open set $U$ around $x$ and any $p$ in $\mathbb{N}$, there are $n$ and $m$ in $\mathbb{N}$ with $n>m>p$ and there is $x^{\prime}$ in $P \cap U$ with $\left|f_{n}\left(x^{\prime}\right)-f_{m}\left(x^{\prime}\right)\right| \geq \varepsilon$.

At a limit ordinal $a$ we set

$$
P_{\varepsilon,\left(f_{n}\right)}^{a}=\bigcap_{\beta<\alpha} P_{\varepsilon,\left(f_{n}\right)}^{\beta} .
$$

Let

$$
\gamma\left(\left(f_{n}\right), \varepsilon\right)=\left\{\begin{array}{l}
\text { the least } a \text { with } K_{\varepsilon,\left(f_{n}\right)}^{a}=\varnothing \text { if such an } a \text { exists, } \\
\omega_{1}, \quad \text { otherwise. }
\end{array}\right.
$$

Define the convergence rank $\gamma\left(\left(f_{n}\right)\right)$ of $\left(f_{n}\right)$ by

$$
\gamma\left(\left(f_{n}\right)\right)=\sup \left\{\gamma\left(\left(f_{n}\right), \varepsilon\right): \varepsilon>0\right\} .
$$

The derivative sets $P_{\varepsilon,\left(f_{n}\right)}^{1}$ are defined by Zalcwasser in [Z], Gillespie and Hurwicz in $[\mathrm{G}-\mathrm{H}]$. The convergence rank is defined by Kechris and Louveau in $[\mathrm{K}-\mathrm{L}]$. 
Remark 1. (i) By compactness of $K$ it is easy to see that $\beta(f, \varepsilon)$ and $\gamma\left(\left(f_{n}\right), \varepsilon\right)$ are isolated ordinals for all positive real number $\varepsilon$.

(ii) As in the proof of Corollary 4, section 2 of $[\mathrm{K}-\mathrm{L}]$, it is easy to prove that $\beta\left(X_{A}\right)=\beta\left(X_{A}, 1 / 2\right)$ and hence $\beta\left(X_{A}\right)$ is an isolated ordinal.

2. Definition ([H-O-R], $[\mathrm{K}-\mathrm{L}])$. Let $K$ be a compact metric space.

(a) $\operatorname{DBSC}(K)$ is the class of differences of two bounded semicontinuous realvalued functions on $K$. Without difficulty it can be shown that $\operatorname{DBSC}(K)$ coincides with the class of those $F: K \rightarrow \mathbb{R}$ for which there exist $\left(f_{n}\right) \subset C(K)$ and $C \in \mathbb{R}$ such that $f_{0}=0, f_{n} \rightarrow F$ pointwise, and $\sum_{n=0}^{\infty}\left|f_{n+1}(y)-f_{n}(y)\right| \leq C$ for all $y \in K$.

(b) We define $|\cdot|_{D}: \operatorname{DBSC}(K) \rightarrow \mathbb{R}$ using (a) as follows: $|F|_{D}$ is the infimum of all positive numbers $C$ satisfying the condition in (a). Then $|\cdot|_{D}$ is a norm and $\operatorname{DBSC}(K)$ with $|\cdot|_{D}$ is a Banach space.

3. Theorem ([K-L], Theorem 1, Section 3$) \cdot \mathbb{B}_{1}^{1}(K)$ is the sup-norm-closure of $\operatorname{DBSC}(K)$.

4. Proposition ([K-L], Lemma 5, Section 2). Let $K$ be a compact metric space, $\left(f_{n}\right),\left(g_{n}\right)$ be the two sequences of functions on $K$, pointwise converging to $f$ and $g$ respectively. If $\xi<\omega_{1}$ is such that $\gamma\left(\left(f_{n}\right)\right) \leq \omega^{\xi}$ and $\gamma\left(\left(g_{n}\right)\right) \leq \omega^{\xi}$, then $\gamma\left(\left(f_{n}+g_{n}\right)\right) \leq \omega^{\xi}$.

5. Theorem ([K-L], Theorem 3, Section 2). Let $\left(f_{n}\right)$ be a bounded sequence of continuous functions on $K$, pointwise converging to some (bounded) Baire-1 function $f$.

Then there exists a sequence $\left(g_{n}\right)$ of convex blocks of $\left(f_{n}\right)$ with $\gamma\left(\left(g_{n}\right)\right)=\beta(f)$.

The following proposition is due to Kechris and Louveau, [K-L], Prop. 9, Section 2 .

6. Proposition. Let $f \in \mathbb{B}_{1}(K), f \geq 0$ and $\xi<\omega_{1}$ with $\beta(f) \leq \omega^{\xi}$ and $n \in \mathbb{N}$, $n>2$. Then there are $n-2$ sets $A_{1}, \ldots, A_{n-2}$ with $\beta\left(X_{A_{k}}\right)<\omega^{\xi}$, such that the function

$$
g=\frac{\|f\|_{\infty}}{n} \sum_{k=1}^{n-2} X_{A_{k}}
$$

satisfies $0 \leq g \leq f \leq g+2\|f\|_{\infty} / n$.

7. Theorem $([\mathrm{K}-\mathrm{N}])$. Let $K$ be a compact metric space, $\xi<\omega$ an ordinal and $f \in \mathbb{B}_{1}(K)$. Then $f \in \mathbb{B}_{1}^{\xi+1}(K)$ if and only if there is a sequence $\left(f_{n}\right) \subset \operatorname{DBSC}(K)$ converging pointwise to $f$ such that $\gamma\left(\left(f_{n}\right)\right) \leq \omega^{\xi}$.

Proof. Necessity. Let $f \in \mathbb{B}_{1}^{\xi+1}(K)$. Then $\beta(f) \leq \omega^{\xi+1}$.

Case 1. We assume that $f=X_{A}$. Then by Remark 1 (ii) $\beta\left(X_{A}\right)$ is isolated and hence $\beta\left(X_{A}\right)<\omega^{\xi+1}$. Then there is $k<\omega$ such that $\beta\left(X_{A}\right)<k \omega^{\xi}$. Then there is a decreasing sequence $\left(F_{\eta}\right)_{\eta<k \omega}$ of closed subsets of $K$ such that

$$
A=\bigcup_{\substack{\eta<k \omega^{\xi} \\ \eta \text { even }}}\left(F_{\eta} \backslash F_{\eta+1}\right) \text {. }
$$

We set

$$
A_{i}=\bigcup\left\{\left(F_{\eta} \backslash F_{\eta+1}\right): i \omega^{\xi} \leq \eta<(i+1) \omega^{\xi}, \eta \text { even }\right\} \quad \forall i=0,1, \ldots, k .
$$


Then $X_{A}=X_{A_{1}}+\cdots+X_{A_{k}}$. By Proposition 4 we shall show the conclusion for $X_{A_{i}}, i=0,1, \ldots, k$.

Without loss of generality we can assume that $k=1$, that is,

$$
A=\bigcup_{\substack{\eta<\omega^{\xi} \\ \eta \text { even }}}\left(F_{\eta} \backslash F_{\eta+1}\right) .
$$

Let $\left\{\eta_{1}, \eta_{2}, \ldots, \eta_{n}, \ldots\right\}$ be an enumeration of the set $\left\{\eta: \eta\right.$ even with $\left.0 \leq \eta<\omega^{\xi}\right\}$. For every $n \in \mathbb{N}$ we set:

$$
A_{n}=\bigcup_{i=1}^{n}\left(F_{\eta_{i}} \backslash F_{\eta_{i}+1}\right) .
$$

Then $X_{A_{n}} \in \operatorname{DBSC}(K)$ for every $n \in \mathbb{N}$ and $X_{A_{n}} \rightarrow X_{A}$ pointwise.

We shall show that: $\gamma\left(\left(X_{A_{n}}\right)\right)<\omega^{\xi}$.

Let $0<\varepsilon<1$. We prove first that $K_{\varepsilon,\left(X_{\left.A_{n}\right)}\right)}^{1} \subset \bigcap_{\eta<\omega} F_{\eta}$.

Let $x \in K_{\varepsilon,\left(X_{A_{n}}\right)}^{1}$ such that $x \notin \bigcap_{\eta<\omega} F_{\eta}$.

Then there exists an open neighborhood $V$ of $x$ such that $\bar{V} \cap \bigcap_{\eta<\omega} F_{\eta}=\varnothing$.

Since $K$ is compact we have that $V$ intersects at most finitely many $\left(F_{\eta}\right)_{\eta<\omega}$. Then since $\left(F_{\eta}\right)_{\eta<\omega}$ is decreasing we have that $V$ intersects at most finite many $F_{\eta_{n}} \backslash F_{\eta_{n}+1}, n=1,2, \ldots$. Hence there is $n_{0} \in \mathbb{N}$ such that $X_{A_{n} \mid V}=X_{A_{n_{0}} \mid V}$ for every $n \geq n_{0}$ which is a contradiction, because $x \in K_{\varepsilon,\left(X_{A_{n}}\right)}^{1}$. By induction $K_{\varepsilon,\left(x_{A_{n}}\right)}^{\eta} \subset \bigcap_{\eta<n \omega} F_{\eta}$ for every $n<\omega$. Then $K_{\varepsilon,\left(X_{A_{n}}\right)}^{\omega} \subset \bigcap_{\eta<\omega^{2}} F_{\eta}$.

Again by induction, we have: $K_{\varepsilon,\left(X_{A_{n}}\right)}^{\omega^{n}} \subset \bigcap_{\eta<\omega^{n+1}} F_{\eta}$ for every $n<\omega$.

Hence $K_{\varepsilon,\left(X_{\left.A_{n}\right)} \omega^{\xi-1}\right.} \subset \bigcap_{\eta<\omega \xi} F_{\eta}$ and since $X_{A_{n}}(y)=0$ for every $y \in \bigcap_{\eta<\omega \xi} F_{\eta}$ and $n \in \mathbb{N}$ we have $K_{\varepsilon,\left(X_{A_{n}}\right)}^{\omega^{\xi-1}+1}=\varnothing$, that is, $\gamma\left(\left(X_{A_{n}}\right)\right)=\omega^{\xi-1}+1<\omega^{\xi}$.

Case 2. Suppose that $f \geq 0$. Then using Theorem 5 we find a sequence $\left(g_{n}\right)$ where $0 \leq g_{n}=\sum_{i=1}^{k_{n}} a_{i}^{n} X_{A_{i}^{n}}$ with $\beta\left(X_{A_{i}^{n}}\right)<\omega^{\xi+1}$ for every $i=1,2, \ldots, k_{n}, n \in \mathbb{N}$, such that:

$$
0 \leq g_{1}+\cdots+g_{n} \leq f \leq g_{1}+\cdots+g_{n}+\frac{\|f\|_{\infty}}{2^{n+2}} \quad \forall n \in \mathbb{N}
$$

Then for every $n>1$ we have

$$
\begin{aligned}
0 & \leq g_{n}=g_{1}+\cdots+g_{n}+\frac{\|f\|_{\infty}}{2^{n+1}}-g_{1}-\cdots-g_{n-1}-\frac{\|f\|_{\infty}}{2^{n+1}} \\
& \leq f+\frac{\|f\|_{\infty}}{2^{n+1}}-f \leq \frac{\|f\|_{\infty}}{2^{n}} .
\end{aligned}
$$

Hence $\left\|g_{n}\right\|_{\infty} \leq\|f\|_{\infty} 2^{-n}$ for any $n>1$. Without loss of generality we can assume that $\|f\|_{\infty} \leq 1$. Then $\left\|g_{n}\right\|_{\infty} \leq 2^{-n}$ for every $n>1$. Also $f=\sum_{n=1}^{\infty} g_{n}$ uniformly.

Since $\beta\left(X_{A_{i}^{n}}\right)<\omega^{\xi+1}$ for every $i=1,2, \ldots, k_{n}, n \in \mathbb{N}$, and by Case 1 and Proposition 4 we have that, for each $n \in \mathbb{N}$, there is $\left(g_{n}^{p}\right) \subset \operatorname{DBSC}(K)$ pointwise converging to $g_{n}$ such that $g_{n}^{p} \geq 0$ for every $p \in \mathbb{N}$ and $\gamma\left(\left(g_{n}^{p}\right)\right) \leq \omega^{\xi}$. For $\xi=0$ this is proved by Kechris and Louveau (cf. [K-L]).

Since $\left\|g_{n}^{p}\right\|_{\infty} \leq\left\|g_{n}\right\|_{\infty} \leq 2^{-n}$ for every $n>1$ and $\left\|g_{1}^{p}\right\|_{\infty} \leq\left\|g_{1}\right\|_{\infty}$ for every $p \in \mathbb{N}$, we have that for any $p \in \mathbb{N} \sum_{n=1}^{\infty} g_{n}^{p}<\infty$ uniformly.

For any $p \in \mathbb{N}$ we set $g^{p}=\sum_{n=1}^{\infty} g_{n}^{p}$. Since $g_{n}^{p} \in \operatorname{DBSC}(K)$ for every $n \in \mathbb{N}$ and the convergence of the series is uniform we have $g^{p} \in \mathbb{B}_{1}^{1}(K)$ for every $p \in \mathbb{N}$. 
Then, by Theorem 3 we have that for every $p \in \mathbb{N}$ there exists $f_{p} \in \operatorname{DBSC}(K)$ such that $\left\|g^{p}-f_{p}\right\|_{\infty}<\frac{1}{p}$. Then since $\left(g^{p}\right)$ is pointwise converging to $f$ we have that and the sequence $\left(f_{p}\right)$ is also pointwise converging to $f$.

The proof of Case 2 can be finished by proving that $\gamma\left(\left(f_{n}\right)\right) \leq \gamma\left(\left(g^{p}\right)\right) \leq \omega^{\xi}$.

We see this, as follows:

Let $\varepsilon>0, P$ be a closed subset of $K$. We shall show that $P_{\varepsilon,\left(f_{p}\right)}^{1} \subset P_{\varepsilon / 2,\left(g^{p}\right)}^{1}$.

Let $x \in P_{\varepsilon,\left(f_{p}\right)}^{1} \backslash P_{\varepsilon / 2,\left(g^{p}\right)}^{1}$. Then there exists an open subset $U$ of $P$ with $x \in U$ and $p_{0} \in \mathbb{N}$ such that

$$
\left|g^{p}\left(x^{\prime}\right)-g^{p^{\prime}}\left(x^{\prime}\right)\right| \leq \varepsilon / 2 \quad \forall x^{\prime} \in U, p, p^{\prime} \geq p_{0} .
$$

Let $p_{1} \geq p_{0}$ with $\frac{2}{p_{1}}<\frac{\varepsilon}{2}$. Then for each $p, p^{\prime} \geq p_{1}$ and $x^{\prime} \in U$ we have

$$
\begin{aligned}
\left|f_{p}\left(x^{\prime}\right)-f_{p^{\prime}}\left(x^{\prime}\right)\right| & \leq\left|f_{p}\left(x^{\prime}\right)-g^{p}\left(x^{\prime}\right)\right|+\left|g^{p}\left(x^{\prime}\right)-g^{p^{\prime}}\left(x^{\prime}\right)\right|+\left|g^{p^{\prime}}\left(x^{\prime}\right)-f_{p^{\prime}}\left(x^{\prime}\right)\right| \\
& <\frac{1}{p}+\frac{\varepsilon}{2}+\frac{1}{p^{\prime}}<\varepsilon,
\end{aligned}
$$

a contradiction since $x \in P_{\varepsilon,\left(f_{p}\right)}^{1}$. Hence $\gamma\left(\left(f_{p}\right)\right) \leq \gamma\left(\left(g^{p}\right)\right)$.

Note that for $q, q^{\prime} \geq p>1$, we have

$$
\left\|g^{q}-g^{q^{\prime}}\right\|_{\infty} \leq\left\|\sum_{n \leq p} g_{n}^{q}-\sum_{n \leq p} g_{n}^{q^{\prime}}\right\|_{\infty}+4.2^{-p} .
$$

Also, $\gamma\left(\left(g_{n}^{q}\right)\right) \leq \omega^{\xi}$ for all $n \in \mathbb{N}$ and by Proposition 4 we have that $\gamma\left(\left(\sum_{n \leq p} g_{n}^{q}\right)\right) \leq$ $\omega^{\xi}$ and hence by $(*)$ this implies that $\gamma\left(\left(g^{q}\right)\right) \leq \omega^{\xi}$.

Case 3. (General case). If $f \in \mathbb{B}_{1}^{\xi+1}(K)$ then $f=f^{+}-f^{-}$where $f^{+}=\max \{f, 0\}$ and $f^{-}=-\min \{f, 0\}$. Then $0 \leq f^{+}, f^{-} \in \mathbb{B}_{1}^{\xi+1}(K)$ and from Case 2 there are sequences $\left(f_{n}^{1}\right),\left(f_{n}^{2}\right)$ in $\operatorname{DBSC}(\bar{K})$ with $\left(f_{n}^{1}\right)$ converging pointwise to $f^{+},\left(f_{n}^{2}\right)$ converging pointwise to $f^{-}, \gamma\left(\left(f_{n}^{1}\right)\right) \leq \omega^{\xi}$ and $\gamma\left(\left(f_{n}^{2}\right)\right) \leq \omega^{\xi}$. Then $f_{n}^{1}-f_{n}^{2} \in$ $\operatorname{DBSC}(K)$ for every $n \in \mathbb{N},\left(f_{n}^{1}-f_{n}^{2}\right)$ converges pointwise to $f$ and by Proposition 4 we have that $\gamma\left(\left(f_{n}^{1}-f_{n}^{2}\right)\right) \leq \omega^{\xi}$.

Sufficiency. Let $\left(f_{n}\right) \subset \operatorname{DBSC}(K)$ be a sequence converging pointwise to $f$ with $\gamma\left(\left(f_{n}\right)\right) \leq \omega^{\xi}$. We prove that $\beta(f) \leq \omega^{\xi}$.

Claim. $P_{\varepsilon, f}^{\omega} \subset P_{\varepsilon / 3,\left(f_{n}\right)}^{1}$ for all closed subsets $P$ of $K$ and $\varepsilon>0$.

[Proof of claim: Let $P$ be a closed subset of $K$ and $x \in P_{\varepsilon, f}^{\omega} \backslash P_{\varepsilon / 3,\left(f_{n}\right)}^{1}$. Then choose an open subset $V$ of $P$ with $x \in V$ and $n_{0} \in \mathbb{N}$ such that

$$
\left|f_{m}(y)-f_{n}(y)\right| \leq \varepsilon / 3 \quad \forall y \in \bar{V}, n \geq n_{0} .
$$

Then $\left|f_{n_{0}}(y)-f_{n}(y)\right| \leq \varepsilon / 3$ for all $y \in \bar{V}$, all $n \geq n_{0}$ and since $\left(f_{n}\right)$ converges pointwise to $f$ we have that $\left|f_{n_{0}}(y)-f(y)\right| \leq \varepsilon / 3$ for all $y \in \bar{V}$.

Then, $\bar{V}_{\varepsilon, f}^{\eta} \subset \bar{V}_{\varepsilon / 3, f_{n_{0}}}^{\eta}$ for all $\eta<\omega$. Since $\beta\left(f_{n_{0}}\right) \leq \omega$ we have $\bar{V}_{\varepsilon / 3, f_{n_{0}}}^{\omega}=\varnothing$.

Then $V \cap P_{\varepsilon, f}^{\omega} \subset \bar{V}_{\varepsilon, f}^{\omega} \subset \bar{V}_{\varepsilon / 3, f_{n_{0}}}^{\omega}=\varnothing$, a contradiction, since $x \in V \cap P_{\varepsilon, f}^{\omega}$. Hence the proof of the claim is finished.]

By induction and applying the claim we get

$$
K_{\varepsilon, f}^{m \omega} \subset K_{\varepsilon / 3,\left(f_{n}\right)}^{m} \quad \forall m<\omega \Rightarrow K_{\varepsilon, f}^{\omega^{2}} \subset K_{\varepsilon / 3,\left(f_{n}\right)}^{\omega} .
$$

Also, by induction we have $K_{\varepsilon, f}^{\omega^{n+1}} \subset K_{\varepsilon / 3,\left(f_{n}\right)}^{\omega^{n}}$ for all $n<\omega$. 
Hence $K_{\varepsilon, f}^{\omega^{\xi+1}} \subset K_{\varepsilon / 3,\left(f_{n}\right)}^{\omega^{\xi}}=\varnothing$ and hence $\beta(f) \leq \omega^{\xi+1}$.

Remark 2. In Theorem 7 , the sequence $\left(f_{n}\right)$ can in fact also be chosen uniformly bounded (as the proof shows).

For $\xi=1$, Theorem 7 was proved by Kechris and Louveau in [K-L].

8. Theorem $([\mathrm{K}-\mathrm{N}])$. Let $K$ be a compact metric space, $f, f_{n} \in \mathbb{B}_{1}(K), n \in \mathbb{N}$, with $\left(f_{n}\right)$ converging pointwise to $f, \lambda<\omega_{1}$ a limit ordinal and $m<\omega$ such that

$$
\sup \left\{\beta\left(f_{n}\right): n \in \mathbb{N}\right\}<\omega^{\lambda} \quad \text { and } \quad \gamma\left(\left(f_{n}\right)\right) \leq \omega^{\lambda+m} .
$$

Then $\beta(f) \leq \omega^{\lambda+m}$.

Proof. Since $\lambda$ is a limit ordinal and $\sup \left\{\beta\left(f_{n}\right): n \in \mathbb{N}\right\}<\omega^{\lambda}$ we choose a strictly increasing sequence $\left(\lambda_{n}\right)$ such that $\sup _{n} \lambda_{n}=\lambda$ and $\sup \left\{\beta\left(f_{n}\right): n \in \mathbb{N}\right\}<\omega^{\lambda_{1}}$.

Claim. $P_{\varepsilon, f}^{\omega^{\lambda_{1}}} \subset P_{\varepsilon / 3,\left(f_{n}\right)}^{1}$ for all closed subsets $P$ of $K$ and $\varepsilon>0$.

[Proof of claim: Let $P \subset K$ be closed, $\varepsilon>0$ and $x \in P_{\varepsilon, f}^{\omega^{\lambda_{1}}} \backslash P_{\varepsilon / 3,\left(f_{n}\right)}^{1}$.

Then there exists an open subset $V$ of $P$ with $x \in V$ and $n_{0} \in \mathbb{N}$ such that

$$
\left|f_{m}(y)-f_{n}(y)\right| \leq \varepsilon / 3 \quad \forall y \in \bar{V}, n, m \geq n_{0} .
$$

Then $\left|f_{n_{0}}(y)-f(y)\right| \leq \varepsilon / 3 \forall y \in \bar{V}, n \geq n_{0}$ and hence $\bar{V}_{\varepsilon, f}^{\eta} \subset \bar{V}_{\varepsilon / 3, f_{n_{0}}}^{\eta} \forall \eta<\omega^{\lambda_{1}}$.

Since $\beta\left(f_{n_{0}}\right) \leq \omega^{\lambda_{1}}$ implies that $\bar{V}_{\varepsilon / 3, f_{n_{0}}}^{\omega^{\lambda_{1}}}=\varnothing$. Also $V \cap P_{\varepsilon, f}^{\omega^{\lambda_{1}}} \subset \bar{V}_{\varepsilon / 3, f_{n_{0}}}^{\omega^{\lambda_{1}}}$.

Then $V \cap P_{\varepsilon, f}^{\omega^{\lambda_{1}}}=\varnothing$, a contradiction. Hence the proof of the claim is finished.]

By induction and applying the claim we get $K_{\varepsilon, f}^{\theta \omega^{\lambda_{1}}} \subset K_{\varepsilon / 3,\left(f_{n}\right)}^{\theta} \forall \theta<\omega^{\lambda}$ and hence $K_{\varepsilon, f}^{\omega^{\lambda}}=\bigcap_{n=1}^{\infty} K_{\varepsilon, f}^{\omega^{\lambda_{n}+\lambda_{1}}} \subset \bigcap_{n=1}^{\infty} K_{\varepsilon / 3,\left(f_{n}\right)}^{\omega^{\lambda_{n}}}=K_{\varepsilon / 3,\left(f_{n}\right)}^{\omega^{\lambda}}$.

By induction we have that $K_{\varepsilon, f}^{n \omega^{\lambda}} \subset K_{\varepsilon / 3,\left(f_{n}\right)}^{n \omega^{\lambda}} \forall n<\omega$ and hence $K_{\varepsilon, f}^{\omega^{\lambda+1}} \subset$ $K_{\varepsilon / 3,\left(f_{n}\right)}^{\omega^{\lambda+1}}$.

Also, by induction we get $K_{\varepsilon, f}^{\omega^{\lambda+m}} \subset K_{\varepsilon / 3,\left(f_{n}\right)}^{\omega^{\lambda+m}}=\varnothing$ and hence $\beta(f) \leq \omega^{\lambda+m}$.

Note. Theorems 7 and 8 are due jointly to Professor Negrepontis (cf. [K-N]). I am grateful to Professor Negrepontis for his kind permission to present some of our joint work here.

In the following corollary it is proved that the conclusion of Theorem 7 is not true for $\xi \geq \omega$.

9. Corollary. Le $K$ be a compact metric space, $\omega \leq \xi<\omega_{1}, f \in \mathbb{B}_{1}(K)$ and $\left(f_{n}\right) \subset \operatorname{DBSC}(K)$ such that $\left(f_{n}\right)$ is pointwise converging to $f$ and $\gamma\left(\left(f_{n}\right)\right) \leq \omega^{\xi}$.

Then $\beta(f) \leq \omega^{\xi}$.

Proof. If $\xi \geq \omega$ there is a limit ordinal $\lambda \geq \omega$ and $m<\omega$ such that $\xi=\lambda+m$. Also $\sup \left\{\beta\left(f_{n}\right): n \in \mathbb{N}\right\}=\omega<\omega^{\omega} \leq \omega^{\lambda}$. Hence by Theorem 8 we have $\beta(f) \leq \omega^{\lambda}$.

10. Proposition. Let $K$ be a scattered compact metric space with $K^{\left(\omega^{\omega+1}\right)} \neq \varnothing$. Then there is a sequence $\left(f_{n}\right) \subset \mathbb{B}_{1}(K), f \in \mathbb{B}_{1}(K)$ such that $\left(f_{n}\right)$ is pointwise converging to $f, \sup \left\{\beta\left(f_{n}\right): n \in \mathbb{N}\right\}=\omega^{\omega}, \gamma\left(\left(f_{n}\right)\right) \leq \omega^{\omega+1}$ and $\beta(f)>\omega^{\omega+1}$.

Proof. We set

$$
A=\bigcup\left\{\left(K^{(\eta)} \backslash K^{(\eta+1)}\right): \eta \text { even and } \eta<\omega^{\omega+1}\right\} .
$$


Then $\beta\left(X_{A}\right)=\omega^{\omega+1}+1$. For every $n \in \mathbb{N}$ we set

$$
\begin{array}{r}
A_{n}^{k}=\bigcup\left\{\left(K^{(\eta)} \backslash K^{(\eta+1)}\right): \eta \text { even and }(k-1) \omega^{\omega} \leq \eta<\omega^{\omega}+\omega^{\eta}\right\}, \\
k=1,2, \ldots, n .
\end{array}
$$

Then we have $\omega^{n}<\beta\left(X_{A_{n}^{k}}\right) \leq \omega^{n+1} \forall k=1,2, \ldots, n, n \in \mathbb{N}$.

We set $A_{n}=\bigcup_{k=1}^{n} A_{n}^{k} \forall n \in \mathbb{N}$. Then $X_{A_{n}}=X_{A_{n}^{1}}+\cdots+X_{A_{n}^{n}}$ and hence $\omega^{n}<\beta\left(X_{A_{n}}\right) \leq \omega^{n+1}$ for all $n \in \mathbb{N}$.

Then $\sup \left\{\beta\left(X_{A_{n}}\right): n \in \mathbb{N}\right\}=\omega^{\omega}$. Also $\left(X_{A_{n}}\right)$ is pointwise converging to $X_{A}$.

The proof will be finished by proving that $\gamma\left(\left(X_{A_{n}}\right)\right) \leq \omega+1$.

To see this, if $\varepsilon>0$ then $K_{\varepsilon,\left(X_{A_{n}}\right)}^{m} \subset \bigcap_{\eta<m \omega} K_{\omega}^{(\eta)}$ for all $m<\omega$ and hence $K_{\varepsilon,\left(X_{A_{n}}\right)}^{\omega} \subset \bigcap_{\eta<\omega} K_{\omega+1}^{(\eta)}$. Since the functions $X_{A_{n}}$ are zero on $\bigcap_{\eta<\omega} K_{\omega+1}^{(\eta)}$ we have that $K_{\varepsilon,\left(X_{A_{n}}\right)}^{\omega+1}=\varnothing$.

Remark 3. Proposition 10 is an example, showing that one of the conditions in Theorem 7 is best possible. Also, there is surely no need to assume $K$ scattered in the statement of the result. I thank the referee for this remark.

11. Proposition $([\mathrm{H}-\mathrm{O}-\mathrm{R}])$. Let $K$ be a compact metric space, $m \in \mathbb{N}, \delta>0$ and a function $f: K \rightarrow \mathbb{R}$ is such that $K_{\varepsilon, f}^{m} \neq \varnothing$. Then $|f|_{D} \geq m \delta / 4$.

12. Proposition. Let $K$ be a compact metric space, $f \in \mathbb{B}_{1}(K), \xi<\omega,\left(f_{n}\right) \subset$ $\operatorname{DBSC}(K)$ pointwise converging to $f, \gamma\left(\left(f_{n}\right)\right) \leq \omega^{\xi}$ and $\sup _{n}\left|f_{n}\right|_{D}<\infty$.

Then $\beta(f) \leq \omega^{\xi}$.

Proof. Let $\varepsilon>0$.

Claim 1. $\exists n_{0} \in \mathbb{N}: \beta\left(f_{n}, \varepsilon / 3\right)=\beta\left(f_{n_{0}}, \varepsilon / 3\right) \forall n \geq n_{0}$.

[Proof of Claim 1. Let then $\beta\left(f_{n}, \varepsilon / 3\right)=m_{n}+1$, where $m_{n}, n \in \mathbb{N}$. Then $K_{\varepsilon, f_{n}}^{m_{n}} \neq \varnothing$ and hence by Proposition 10 we have that $\left|f_{n}\right|_{D} \geq m_{n} \varepsilon / 12$. If the sequence $\left(m_{n}\right)$ is infinite then $\sup _{n}\left|f_{n}\right|_{D}=\infty$, a contradiction.

Thus there is $n_{0} \in \mathbb{N}$ such that $m_{n}=m_{n_{0}}$ for all $n \geq n_{0}$.]

Claim 2. If $m=\beta\left(f_{n_{0}}, \varepsilon / 3\right)$ then $P_{\varepsilon, f}^{m} \subset P_{\varepsilon / 3,\left(f_{n}\right)}^{\prime}$ for each closed subset $P$ of $K$.

[Proof of Claim 2. Let $x \in P_{\varepsilon, f}^{m} \backslash P_{\varepsilon / 3,\left(f_{n}\right)}^{\prime}$. Then there are an open neighborhood $V$ of $x$ in $P$ and $n_{0} \in \mathbb{N}$ such that

$$
\left|f_{m}(y)-f_{n}(y)\right| \leq \varepsilon / 3 \quad \forall n, m \geq n_{0}, y \in \bar{V} .
$$

Then, $\left|f_{n_{0}}(y)-f(y)\right| \leq \varepsilon / 3$ for all $y \in \bar{V}$ and hence $\bar{V}_{\varepsilon, f}^{\prime} \subset \bar{V}_{\varepsilon / 3, f_{n_{0}}}^{\prime}$.

Finally, by induction we get $\bar{V}_{\varepsilon, f}^{m} \subset \bar{V}_{\varepsilon / 3, f_{n_{0}}}^{m}=\varnothing$. Since $V \cap P_{\varepsilon, f}^{m} \subset \bar{V}_{\varepsilon, f}^{m}$ we have $V \cap P_{\varepsilon, f}^{m}=\varnothing$, a contradiction since $x \in V \cap P_{\varepsilon, f}^{m}$.]

Since $\gamma\left(\left(f_{n}\right)\right) \leq \omega^{\xi}$ we have that $\gamma\left(\left(f_{n}\right), \varepsilon / 3\right)<\omega^{\xi}$ and hence there is $k<\omega$ such that $\gamma\left(\left(f_{n}\right), \varepsilon / 3\right)<k \omega^{\xi-1}$. Applying Claim 1 we have

$$
K_{\varepsilon, f}^{m} \subset K_{\varepsilon / 3,\left(f_{n}\right)}^{\prime}, K_{\varepsilon, f}^{2 m} \subset K_{\varepsilon / 3,\left(f_{n}\right)}^{\prime \prime}, \ldots, K_{\varepsilon, f}^{m k \omega^{\xi-1}} \subset K_{\varepsilon / 3,\left(f_{n}\right)}^{k \omega^{\xi-1}}=\varnothing .
$$

Then $\beta(f, \varepsilon) \leq k m \omega^{\xi-1}<\omega^{\xi}$. Hence it is proved that $\beta(f) \leq \omega^{\xi}$.

13. Definition $([\mathrm{H}-\mathrm{O}-\mathrm{R}])$. Define $\mathbb{B}_{1 / 4}(K)$ to be the set of those $f$ in $\mathbb{B}_{1}(K)$ for which there is a sequence $\left(f_{n}\right)$ in $\operatorname{DBSC}(K)$ that converges uniformly to $f$ and is such that $\sup _{n}\left|f_{n}\right|_{D}<\infty$. 
14. Theorem ([H-O-R], Th. 6.1). Let $K$ be a compact metric space and let $f \in$ $\mathbb{B}_{1}(K)$. Then $f \in \mathbb{B}_{1 / 4}(K)$ iff there exists a $C<\infty$ such that for all $\varepsilon>0$ there exists a sequence $\left(s_{n}\right)_{n=0}^{\infty} \subset C(K), s_{0}=0$, with $\left(s_{n}\right)$ converging pointwise to $f$ and such that for all subsequences $\left(n_{i}\right)$ of $\{0\} \cup \mathbb{N}$ and $x \in K$,

$$
\sum_{j \in B\left(\left(n_{i}\right), x\right)}\left|s_{n_{j+1}}(x)-s_{n_{j}}(x)\right| \leq C,
$$

where $B\left(\left(n_{i}\right), x\right)=\left\{j:\left|s_{n_{j+1}}(x)-s_{n_{j}}(x)\right| \geq \varepsilon\right\}$.

The above result gave the idea for the definition of the rank $\delta$ (cf. [K-N]). I am grateful to Professor Negrepontis who gave me this idea.

15. Definition. Let $K$ be a compact metric space, $f, s_{n}: K \rightarrow \mathbb{R}, n \in \mathbb{N}$, real-valued functions with $s_{0}=0$ such that $\left(s_{n}\right)$ is pointwise converging to $f$. For each closed subset $P$ of $K$ and $\varepsilon>0$ we set:

$$
\begin{aligned}
& P^{0}\left(\left(s_{n}\right), \varepsilon\right)=P, \\
& P^{\prime}\left(\left(s_{n}\right), \varepsilon\right)=\left\{\begin{aligned}
& x \in P: \forall 0<C<\infty, \forall m \in \mathbb{N}, \forall U \subset K \text { open neighborhood of } x \\
& \exists j_{p}>\cdots>j_{1} \geq m \text { and } x^{\prime} \in U \cap P \text { such that } \\
&\left|s_{j_{i+1}}\left(x^{\prime}\right)-s_{j_{i}}\left(x^{\prime}\right)\right|>\varepsilon \text { for } i=1,2, \ldots, p
\end{aligned}\right. \\
& \text { and } \left.\sum_{i=1}^{p}\left|s_{j_{i+1}}\left(x^{\prime}\right)-s_{j_{i}}\left(x^{\prime}\right)\right|>C\right\} .
\end{aligned}
$$

For each ordinal $a<\omega_{1}$ we set

$$
P^{a+1}\left(\left(s_{n}\right), \varepsilon\right)=\left(P^{a}\left(\left(s_{n}\right), \varepsilon\right)\right)^{\prime}\left(\left(s_{n}\right), \varepsilon\right)
$$

If $\beta$ is a limit ordinal, we set

$$
P^{\beta}\left(\left(s_{n}\right), \varepsilon\right)=\bigcap_{a<\beta} P^{a}\left(\left(s_{n}\right), \varepsilon\right) .
$$

We set

$$
\delta\left(\left(s_{n}\right), \varepsilon\right)=\left\{\begin{array}{l}
\text { the least ordinal } a<\omega_{1} \text { such that } K^{a}\left(\left(s_{n}\right), \varepsilon\right)=\varnothing \\
\quad \text { if such an } a \text { exists } \\
\omega_{1}, \quad \text { otherwise. }
\end{array}\right.
$$

and

$$
\delta\left(\left(s_{n}\right)\right)=\sup \left\{\delta\left(\left(s_{n}\right), \varepsilon\right): \varepsilon>0\right\} .
$$

Remark 4. $\delta\left(\left(s_{n}\right)\right) \leq \gamma\left(\left(s_{n}\right)\right)$.

We see this as follows: Let $P$ be a closed subset of $K, \varepsilon>0$ and $x \in P \backslash P_{\varepsilon,\left(s_{n}\right)}^{1}$. Then there are an open neighborhood of $x$ in $P$ and $p \in \mathbb{N}$ such that for every $y \in U$ and $m, n \in \mathbb{N}$ with $m, n \geq p$ we have $\left|f_{m}(y)-f_{n}(y)\right| \leq \varepsilon$. By definition of $P^{\prime}\left(\left(s_{n}\right), \varepsilon\right)$ we have that $x \notin P^{\prime}\left(\left(s_{n}\right), \varepsilon\right)$. Hence $P^{\prime}\left(\left(s_{n}\right), \varepsilon\right) \subset P_{\varepsilon,\left(s_{n}\right)}^{1}$.

16. Proposition $([\mathrm{H}-\mathrm{O}-\mathrm{R}])$. Let $X$ be a Banach space and $C, D$ be convex subsets of $X$. Then

$$
\inf \{\|c-d\|: c \in C, d \in D\}=\inf \{\|c-d\|: c \in \widetilde{C}, d \in \widetilde{D}\},
$$

where $\widetilde{C}$ and $\widetilde{D}$ are the $w^{*}$-closure of $C$ and $D$ in $X^{* *}$. 
17. Theorem. Let $K$ be a compact metric space, $f \in \mathbb{B}_{1}(K)$, a sequence $\left(f_{n}\right) \subset$ $C(K)$ pointwise converging to $f$ and $\xi<\omega_{1}$.

Then the following equivalences are satisfied:

(i) If $1 \leq \xi<\omega$, then $\beta(f) \leq \omega^{\xi}$ if and only if there exists a sequence $\left(s_{n}\right)$ of convex blocks of $\left(f_{n}\right)$ with $\delta\left(\left(s_{n}\right)\right) \leq \omega^{\xi-1}$.

(ii) If $\xi \geq \omega$, then $\beta(f) \leq \omega^{\xi}$ if and only if there exists a sequence $\left(s_{n}\right)$ of convex blocks of $\left(f_{n}\right)$ with $\delta\left(\left(s_{n}\right)\right) \leq \omega^{\xi}$.

Proof. (i). Necessity. Let $1 \leq \xi<\omega$ and $\beta(f) \leq \omega^{\xi}$. Then by Theorem 7 we have there is a sequence $\left(F_{n}\right) \subset \operatorname{DBSC}(K)$ pointwise converging to $f$ and $\gamma\left(\left(F_{n}\right)\right) \leq \omega^{\xi}$. Let $\varepsilon>0$. Then $\gamma\left(\left(F_{n}\right), \frac{\varepsilon}{4}\right)=\theta+1<\omega^{\xi}$.

For every $\eta \leq \theta$ we set $K_{\eta}=K_{\varepsilon / 4,\left(F_{n}\right)}^{\eta}$. Then for every $\eta \leq \theta$ and $x \in K_{\eta} \backslash K_{\eta+1}$ there are an open neighborhood $U_{x, \eta}$ of $x$ in $K_{\eta}$ and $n \in \mathbb{N}$ such that $\left|F_{n}(y)-f(y)\right| \leq$ $\varepsilon / 4$ for every $y \in \bar{U}_{x, \eta}$. Since $K$ is a compact metric space we have that for every $\eta \leq \theta$ there exists a countable subset $\left\{U_{\eta, k}: k \in \mathbb{N}\right\}$ of $\left\{U_{x, \eta}: x \in K_{\eta} \backslash K_{\eta+1}\right\}$ such that

$$
\bigcup\left\{U_{\eta, k}: k \in \mathbb{N}\right\}=\bigcup\left\{U_{x, \eta}: x \in K_{\eta} \backslash K_{\eta+1}\right\} .
$$

Let $\left\{U_{\eta_{i}, k_{i}}: i \in \mathbb{N}\right\}$ be an enumeration of $\left\{U_{\eta, k}: \eta \leq \theta, k \in \mathbb{N}\right\}$. Then for every $i \in \mathbb{N} \exists n_{1} \in \mathbb{N}$ such that

$$
\left\|f-F_{n_{i}}\right\|_{\bar{U}_{\eta_{i}, k_{i}}}<\varepsilon / 4 .
$$

(If $M$ is a subspace of $K$ we set \|\|$_{M}$ the supremum norm on $C(M)$.)

For every $i \in \mathbb{N}$ let $\left(f_{m}^{i}\right)_{m=0}^{\infty} \subset C(K)$ with $f_{0}^{i}=0,\left(f_{m}^{i}\right)_{m=0}^{\infty}$ is pointwise converging to $F_{n_{i}}$ and

$$
\sum_{m=0}^{\infty}\left|f_{m}^{i}(y)-f_{m}^{i}(y)\right| \leq\left|F_{n_{i}}\right|_{D} \quad \forall y \in \bar{U}_{\eta_{i}, k_{i}} .
$$

By $(*)$ and Proposition 16 we have that there exist a sequence $\left(g_{m}^{1}\right)$ of convex blocks of $\left(f_{m}\right)$ and a sequence $\left(h_{m}^{1}\right)$ of convex blocks of $\left(f_{m}^{1}\right)$ such that

$$
\left\|g_{m}^{1}-h_{m}^{1}\right\|_{\bar{U}_{\eta_{1}, k_{1}}}<\varepsilon / 4 \quad \forall m \in \mathbb{N} .
$$

Then for every $m_{1}, \ldots, m_{p} \in \mathbb{N}$ with $m_{1}<\cdots<m_{\rho}$ and $y \in \bar{U}_{\eta_{1}, k_{1}}$ with $\mid g_{m_{i+1}}^{1}(y)-$ $g_{m_{i}}^{1}(y) \mid \geq \varepsilon$ for all $i=1, \ldots, \rho$ we have

$(* * *) \quad \sum_{i=1}^{\rho}\left|g_{m_{i+1}}^{1}(y)-g_{m_{i}}^{1}(y)\right| \leq \sum_{j=0}^{\infty}\left|f_{j+1}^{1}(y)-f_{j}^{1}(y)\right|+\frac{\varepsilon}{2} \frac{2}{\varepsilon}\left|F_{n_{1}}\right|_{D} \leq 2\left|F_{n_{1}}\right|_{D}$.

[We see this as follows: Let $p, q \in \mathbb{N}$ and $y \in \bar{U}_{\eta_{1}, k_{1}}$ with $\left|g_{p}^{1}(y)-g_{q}^{1}(y)\right| \geq \varepsilon$.

Then by $(* *)$ we have

$$
\varepsilon \leq\left|g_{p}^{1}(y)-g_{q}^{1}(y)\right| \leq \frac{\varepsilon}{2}+\left|h_{p}^{1}(y)-h_{q}^{1}(y)\right| \Rightarrow\left|h_{p}^{1}(y)-h_{q}^{1}(y)\right| \geq \frac{\varepsilon}{2} .
$$

Also, $\sum_{i=1}^{\rho}\left|g_{m_{i+1}}^{1}(y)-g_{m_{i}}^{1}(y)\right| \leq \sum_{j=0}^{\infty}\left|f_{j+1}^{1}(y)-f_{j}^{1}(y)\right|+\frac{\varepsilon}{2} \rho \leq\left|F_{n_{1}}\right|_{D}+\frac{\varepsilon}{2} \rho$. By (1) we have

$$
\rho \frac{\varepsilon}{2} \leq \sum_{i=1}^{\rho}\left|h_{m_{i+1}}^{1}(y)-h_{m_{i}}^{1}(y)\right| \leq\left|F_{n_{1}}\right|_{D} \Rightarrow \rho \leq \frac{2}{\varepsilon}\left|F_{n_{1}}\right|_{D} .
$$

Hence the proof of $(* * *)$ is finished.] 
By induction, for every $i \in \mathbb{N}$ we get a sequence $\left(g_{m}^{i+1}\right)$ of convex blocks of $\left(g_{m}^{i}\right)$ such that $\forall \rho \in \mathbb{N}, m_{1}, \ldots, m_{\rho} \in \mathbb{N}$ with $m_{1}<\cdots<m_{\rho}$ and $y \in \bar{U}_{\eta_{i}, k_{i}}$ with $\left|g_{m_{j+1}}^{i+1}(y)-g_{m_{j}}^{i+1}(y)\right| \geq \varepsilon$ for all $j=1, \ldots, \rho$ we have

$$
\sum_{j=1}^{\rho}\left|g_{m_{j+1}}^{i+1}(y)-g_{m_{j}}^{i+1}(y)\right| \leq 2\left|F_{n_{i+1}}\right|_{D}
$$

We set $s_{0}=0$ and $s_{n}=g_{n}^{n}$ for all $n \in \mathbb{N}$. Then $\left(s_{n}\right)$ is pointwise converging to $f$ and $K^{\eta}\left(\left(s_{n}\right), \varepsilon\right) \subset K_{\eta}$ for all $\eta \leq \theta+1$. Hence $K^{\theta+1}\left(\left(s_{n}\right), \varepsilon\right)=\varnothing$ and hence $\delta\left(\left(s_{n}\right)\right) \leq \omega^{\xi-1}$.

Sufficiency. Let $\delta\left(\left(s_{n}\right)\right) \leq \omega^{\xi-1}$. We shall show that $\gamma\left(\left(s_{n}\right)\right) \leq \omega^{\xi}$ and since $\beta(f) \leq \gamma\left(\left(s_{n}\right)\right)$ we have that $\beta(f) \leq \omega^{\xi}$. Hence we shall show that $P_{\varepsilon,\left(s_{n}\right)}^{\omega} \subset$ $P^{\prime}\left(\left(s_{n}\right), \varepsilon / 2\right)$ for all closed subsets $P$ of $K$.

Let $P$ be a closed subset of $K$ and let $x \in P_{\varepsilon,\left(s_{n}\right)}^{\omega} \backslash P^{\prime}\left(\left(s_{n}\right), \varepsilon / 2\right)$. Then there are a positive real number $C$, an open neighborhood $U$ of $x$ in $P$ and $m \in \mathbb{N}$ such that $\forall p \in \mathbb{N}, n_{1}, \ldots, n_{p} \in \mathbb{N}$ with $n_{p}>\cdots>n_{1} \geq m$ and $y \in U$ with $\left|s_{n_{i+1}}(y)-s_{n_{i}}(y)\right| \geq \varepsilon / 2$ for all $i=1, \ldots, p$ we have $\sum_{i=1}^{p}\left|s_{n_{i+1}}(y)-s_{n_{i}}(y)\right| \leq C$.

Then $p<\frac{C}{\varepsilon}$. Let $n \in \mathbb{N}$ with $n>\frac{C}{\varepsilon}$. Then $x \in P_{\varepsilon,\left(s_{n}\right)}^{n}$. We shall show that there are $y \in U$ and $m_{1}, \ldots, m_{n+1} \in \mathbb{N}$ with $m_{n+1}>\cdots>m_{1} \geq m$ such that $\left|s_{m_{j+1}}(y)-s_{m_{j}}(y)\right|>\varepsilon / 2$ for all $j=1, \ldots, n$, and we shall terminate in a contradiction.

We see this as follows:

$$
\begin{gathered}
x \in P_{\varepsilon,\left(s_{n}\right)}^{n} \cap U \Rightarrow \exists x_{1} \in P_{\varepsilon,\left(s_{n}\right)}^{n-1} \cap U \text { and } m_{1}, m_{2} \in \mathbb{N} \text { with } m_{2}>m_{1} \geq m \text { and } \\
\left|s_{m_{2}}\left(x_{1}\right)-s_{m_{1}}\left(x_{1}\right)\right|>\varepsilon>\varepsilon / 2 .
\end{gathered}
$$

We set $V_{1}=\left\{y \in U:\left|s_{m_{2}}(y)-s_{m_{1}}(y)\right|>\varepsilon / 2\right\}$. $V_{1}$ is open and $x_{1} \in V_{1} \cap P_{\varepsilon,\left(s_{n}\right)}^{n-1}$; hence $\exists x_{2} \in P_{\varepsilon,\left(s_{n}\right)}^{n-2} \cap V_{1}$ and $m_{3} \in \mathbb{N}$ such that $m_{3}>m_{2}$ and $\left|s_{m_{3}}\left(x_{2}\right)-s_{m_{2}}\left(x_{2}\right)\right|>$ $\varepsilon / 2$ (since if $\left|s_{m}(y)-s_{m_{2}}(y)\right| \leq \varepsilon / 2$ for every $m \geq m_{2}$ and $y \in P_{\varepsilon,\left(s_{n}\right)}^{n-2} \cap V_{1}$, then $\left|s_{m}(y)-s_{k}(y)\right| \leq \varepsilon$ for all $m, k \geq m_{2}$ and $y \in P_{\varepsilon,\left(s_{n}\right)}^{n-2} \cap V_{1}$, that is, $x_{1} \notin P_{\varepsilon,\left(s_{n}\right)}^{n-1}$ which is a contradiction).

We set $V_{2}=\left\{y \in V_{1}:\left|s_{m_{3}}(y)-s_{m_{2}}(y)\right|>\varepsilon / 2\right\} . V_{2}$ is open in $P$ and $x_{2} \in V_{2} \subset$ $V_{1}$.

By induction we get $m_{1}, \ldots, m_{n} \in \mathbb{N}$ with $m_{n}>\cdots>m_{1} \geq m, V_{1}, \ldots, V_{n-1}$ open subsets of $P$ with $V_{n-1} \subset \cdots \subset V_{1} \subset U$ and $x_{1} \in P_{\varepsilon,\left(s_{n}\right)}^{n-1} \cap V_{i-1}$ for all $i=1, \ldots, n$ (where $V_{0}=U$ ) such that $\left|s_{m_{i+1}}(y)-s_{m_{i}}(y)\right|>\varepsilon / 2$ for all $y \in V_{i}$, $i=1, \ldots, n-1$. We set $V_{n}=\left\{y \in V_{n-1}:\left|s_{m_{n}}(y)-s_{m_{n-1}}(y)\right|>\varepsilon / 2\right\}$. $V_{n}$ is open in $P$ and $x_{n-1} \in P_{\varepsilon,\left(s_{n}\right)}^{\prime} \cap V_{n}$; hence there is $y \in V_{n}$ and $m_{n+1}>m_{n}$ such that $\left|s_{m_{n+1}}(y)-s_{m_{n}}(y)\right|>\varepsilon / 2$.

Then $\left|s_{m_{j+1}}(y)-s_{m_{j}}(y)\right|>\varepsilon / 2$ for all $j=1, \ldots, n$. Hence the proof of (i) is finished.

(ii) Necessity. By Theorem 5 we have that if $f \in \mathbb{B}_{1}(K)$ with $\beta(f) \leq \omega^{\xi}$ then there is a sequence $\left(s_{n}\right)$ of convex blocks of $\left(f_{n}\right)$ with $\gamma\left(\left(s_{n}\right)\right) \leq \omega^{\xi}$.

Then by Remark 4 we get a conclusion.

Sufficiency. As in (i) we prove that $P_{\varepsilon,\left(s_{n}\right)}^{\omega} \subset P^{\prime}\left(\left(s_{n}\right), \varepsilon / 2\right)$ for all closed subsets $P$ of $K$ and $\varepsilon>0$. Then by induction we get $K_{\varepsilon,\left(s_{n}\right)}^{\omega^{n+1}} \subset K^{\omega^{n}}\left(\left(s_{n}\right), \varepsilon / 2\right)$ for all 
$n \in \mathbb{N}$ and hence $K_{\varepsilon,\left(s_{n}\right)}^{\omega^{\omega}} \subset K^{\omega^{\omega}}\left(\left(s_{n}\right), \varepsilon / 2\right)$. Finally, by induction we get $K_{\varepsilon,\left(s_{n}\right)}^{\omega^{\xi}} \subset$ $K^{\omega^{\xi}}\left(\left(s_{n}\right), \varepsilon / 2\right)$ for all $\varepsilon>0$.

Remark 5. If $\left(s_{n}\right)$ is a sequence of continuous real-valued functions on $K$ with $\delta\left(\left(s_{n}\right)\right)<\omega_{1}$, then $\left(s_{n}\right)$ converges pointwise.

[We see this is follows: As is proved in the demonstration of the sufficiency of Theorem 17 (i) we have that $P_{\varepsilon,\left(s_{n}\right)}^{\omega} \subset P^{\prime}\left(\left(s_{n}\right), \varepsilon / 2\right)$ for all closed subsets $P$ of $K$ and hence

$$
K_{\varepsilon,\left(s_{n}\right)}^{\omega^{\xi+1}} \subset K^{\omega^{\xi}}\left(\left(s_{n}\right), \varepsilon / 2\right) \text { for all } \xi<\omega_{1} .
$$

Assume that $\delta\left(\left(s_{n}\right)\right)<\omega_{1}$. Then there is a $\xi<\omega_{1}$ such that $\delta\left(\left(s_{n}\right)\right)<\omega^{\xi}$; hence $K^{\omega^{\xi}}\left(\left(s_{n}\right), \varepsilon\right)=\varnothing$ for all $\varepsilon>0$ and thus $K_{\varepsilon,\left(s_{n}\right)}^{\omega^{\xi+1}}=\varnothing$ for all $\varepsilon>0$. Then $\gamma\left(\left(s_{n}\right), \varepsilon\right)<\omega^{\xi+1}$ for all $\varepsilon>0$; hence $\gamma\left(\left(s_{n}\right)\right) \leq \omega^{\xi+1}<\omega_{1}$ and thus the sequence $\left(s_{n}\right)$ converges pointwise (cf. [K-L]).]

I thank the referee for this remark.

\section{ACKNOWLEDGMENT}

The results of this paper form a portion of my Doctoral Dissertation. I would like to thank Professor Stylianos Negrepontis, my adviser, for suggesting this problem to me and for many helpful conversations about the matter treated here. Also I would like to thank the referee for his (her) kind corrections and helpful comments.

\section{REFERENCES}

[G-H] D. C. Gillespie and H. A. Hurwicz, On sequences of continuous functions having continuous limits, Trans. Amer. Math. Soc. 32 (1930), 527-543.

[H-O-R] R. Haydon, E. Odell and H. P. Rosenthal, Certain subclasses of Baire-1 functions with Banach space applications, Longhorn Notes, University of Texas at Austin Functional Analysis Seminar 1987-89.

[K-L] A. S. Kechris and A. Louveau, A classification of Baire class 1 functions, Trans. Amer. Math. Soc. 318 (1990), 209-236. MR 90f:26005

[K-N] P. Kiriakouli and S. Negrepontis, A classification of Baire-1 functions, unpublished.

[Z] Z. Zalcwasser, Sur une propriete du champ des fonctions continues, Studia Math. 2 (1930), 63-67.

Department of Mathematics, University of Athens, Panepistimiopolis 15784, Athens, Greece 